\title{
BMJ Open Acupuncture for postoperative pain in laparoscopic surgery: a systematic review protocol
}

\author{
Seunghoon Lee, ${ }^{1}$ Jimin Park, ${ }^{1}$ Jihye Kim, ${ }^{1}$ Jung Won Kang, ${ }^{1}$ Do-Young Choi, ${ }^{1}$ \\ Sun Jin Park, ${ }^{2}$ Dongwoo Nam, ${ }^{1}$ Jae-Dong Lee ${ }^{1}$
}

To cite: Lee S, Park J, Kim J, et al. Acupuncture for postoperative pain in laparoscopic surgery: a systematic review protocol. BMJ Open 2014;4:e006750. doi:10.1136/bmjopen-2014006750

- Prepublication history and additional material for this paper is available online. To view these files please visit the journal online (http://dx.doi.org/10.1136/ bmjopen-2014-006750)

Received 30 September 2014 Revised 28 November 2014 Accepted 28 November 2014

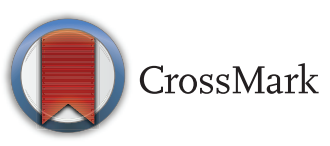

${ }^{1}$ Department of Acupuncture \& Moxibustion, College of Korean Medicine, Kyung Hee University, Seoul, South Korea

${ }^{2}$ Department of Surgery, Kyung Hee University School of Medicine, Seoul, South Korea

\section{Correspondence to}

Professor Jae-Dong Lee;

ljdacu@gmail.com

\section{ABSTRACT}

Introduction: This review aims to evaluate the effectiveness and safety of acupuncture for patients with postoperative pain after laparoscopic surgery.

Methods and analysis: We will search the following databases from their inception to October 2014: MEDLINE, EMBASE, the Cochrane Central Register of Controlled Trials (CENTRAL), the Cumulative Index to Nursing and Allied Health Literature (CINAHL), the Allied and Complementary Medicine Database (AMED), three Chinese databases (China National Knowledge Infrastructure (CNKI), the Chongqing VIP Chinese Science and Technology Periodical Database (VIP) and the Wanfang database), one Japanese database (Japan Science and Technology Information Aggregator, Electronic (J-STAGE)) and eight Korean databases (Korean Association of Medical Journal Edition, Korean Medical Database, Korean Studies Information Service System, National Discovery for Science Leaders, Database Periodical Information Academic, Korean National Assembly Digital Library, Oriental Medicine Advanced Searching Integrated System and Korean Traditional Knowledge Portal). All randomised controlled trials of acupuncture for postoperative pain after laparoscopic surgery will be considered for inclusion. The risk of bias and reporting quality will be assessed using the Cochrane risk of bias tool, the Consolidated Standards of Reporting Trials (CONSORT) and the revised STandards for Reporting Interventions in Clinical Trials of Acupuncture (STRICTA). The risk ratio for dichotomous data and mean difference or standard mean difference for continuous data will be calculated with $95 \% \mathrm{Cls}$.

Dissemination: The results of this review will be disseminated through peer-reviewed publication or conference presentation. Our findings will summarise the current evidence of acupuncture to treat postoperative pain after laparoscopic surgery, and may provide important guidance for acupuncture usage after laparoscopic surgery for clinicians and patients.

Trial registration number: PROSPERO 2014: CRD42014010825.

\section{INTRODUCTION}

\section{Description of the condition}

Laparoscopic surgery is a specialised technique that allows a surgeon to examine the inside of an abdomen or a pelvis using a

\section{Strengths and limitations of this study}

- To the best of our knowledge, this will be the first systematic review to assess the effectiveness and safety of acupuncture for patients with postoperative pain after laparoscopic surgery.

- The trial selection, data extraction, and assessment of risk of bias and reporting quality will be conducted independently by three review authors.

- The risk of bias and the reporting quality might be poor in small studies with high heterogeneity, which might limit the quality of evidence.

miniature video camera, known as a laparoscope, through small incisions in the skin, known as ports. ${ }^{2}$ Laparoscopic surgery has advantages over traditional open surgery in terms of reduced postoperative pain, shorter hospital stay, faster recovery time, decreased postoperative ileus, reduced scarring and preserved immune function; however, laparoscopic surgery still results in substantial postoperative pain around the incision site. ${ }^{3} 4$ Moreover, inflation with carbon dioxide during the surgical procedure frequently induces laparoscopy-induced shoulder pain, secondary to irritation of the phrenic nerve. ${ }^{5}$

\section{Description of the intervention}

Acupuncture is defined as an intervention that stimulates acupuncture points using needles with various manipulations to achieve balance of Qi, which is known as a 'natural energy' that forms part of any living thing. ${ }^{7}$ Acupuncture has been widely used for various forms of musculoskeletal pain in eastern Asian countries, and its effectiveness against chronic pain has been supported by rigorous testing. ${ }^{8}$ Recently, as an adjuvant therapy to conventional anaesthetics after surgery, acupuncture has been used for reducing postoperative pain and analgesic consumption. Some systematic reviews (SRs) reported that acupuncture has an analgesic effect after various types of surgery. ${ }^{9} 10$ 


\section{How the intervention might work}

The mechanism of acupuncture analgesia for postoperative pain remains unclear. Some studies proposed mechanisms of acupuncture through local vasodilation and circulation, ${ }^{11}{ }^{12}$ segmental analgesia based on the gate-control theory of pain, ${ }^{11}$ descending inhibitory pain control by serotonin and norepinephrine, ${ }^{13}$ and the release of opioid peptides including $\beta$-endorphins, enkephalins and dynorphins. ${ }^{14}$

\section{Why it is important to perform this review}

Laparoscopic surgery is widely used, and its use is increasing; laparoscopic cholecystectomy is considered the 'gold standard' treatment option for cholelithiasis. ${ }^{15}$ The amount and duration of opioid consumption after laparoscopic surgery are significantly less than after open surgery. ${ }^{16}$ However, adverse events (eg, nausea/vomiting, pruritus, urinary retention or sedation) induced by opioid analgesics including morphine, meperidine, hydromorphone or fentanyl still frequently bother patients who have undergone laparoscopic surgery and delay hospital discharge. ${ }^{17}$ Moreover, some studies reported that only $60 \%$ of patients were satisfied with postoperative pain control $^{19}$ and about $30-40 \%$ of discharged patients suffered from moderate to severe pain after laparoscopic surgery. ${ }^{18}$ Therefore, it is worth evaluating whether acupuncture, which is known to be safe and also to have analgesic effects, actually reduces postoperative pain and opioid consumption after laparoscopic surgery.

\section{OBJECTIVES}

This study aims to review the evidence for effectiveness and safety of acupuncture for postoperative pain after laparoscopic surgery.

\section{METHODS}

\section{Study registration}

The protocol of review methods has been registered prospectively (CRD42014010825; http://www.crd.york.ac. uk/PROSPERO).

\section{Criteria for including studies in this review \\ Types of studies}

Randomised controlled trials (RCTs) of acupuncture treatment for postoperative pain after laparoscopic surgery will be included for review. Non-RCTs, uncontrolled clinical trials (eg, case studies) and qualitative studies will be excluded. We will not restrict study eligibility according to language or publication.

\section{Types of participants}

All patients undergoing any kind of laparoscopic surgery will be included. When trials also included patients who received other surgeries (eg, abdominal open surgery, haemorrhoidectomy or dental surgery) as well as laparoscopic surgery, we will analyse only the data from the laparoscopic surgery.

\section{Types of interventions}

Acupuncture treatment using needling with various types of stimulation (eg, manual, electroacupuncture or warm acupuncture) on specific points (eg, traditional acupuncture points or tender points including incision regions) will be included. However, we will not include trials in which non-penetrating stimulation on specific points (eg, acupressure, magnets, moxibustion, transcutaneous electrical nerve stimulation or laser therapy) was used.

For control intervention, we will consider no treatment/waiting list, placebo/sham acupuncture and active treatment (eg, central regional opioid analgesia, patientcontrolled analgesia with systemic opioids and peripheral regional analgesic techniques including local anaesthetic infiltration). ${ }^{20}$ However, trials in which acupuncture was compared with other forms of acupuncture or herbal medicine will be excluded. When the acupuncture group received acupuncture and active treatment simultaneously, we will include only trials in which the same active treatment was administered to the acupuncture and control groups.

\section{Types of outcome measures}

\section{Primary outcomes}

1. Pain: relevant overall postoperative pain using any scale (eg, visual analogue scale $(0-100 \mathrm{~mm}$ or $0-10 \mathrm{~cm}$ ) or numerical rating scale) will be analysed. If pain at various sites is reported separately, we will classify and analyse the pain according to the following three groups: (1) parietal pain caused by skin incision (somatic pain); (2) deep intra-abdominal pain originating from internal organs (visceral pain) and (3) shoulder pain due to phrenic nerve irritation ( presumably referred visceral pain). ${ }^{21} 22$

2. Analgesic consumption.

\section{Secondary outcomes}

1. Opioid-related side effects (eg, nausea, vomiting, dizziness or pruritus).

2. Quality of life (QoL): assessed using validated scales (eg, 36-item Short-Form (SF-36) or Euro-QoL).

3. Duration of hospital stay.

4. Time to return to normal activity.

5. Adverse events related to acupuncture treatment.

\section{Search methods for identification of studies}

\section{Electronics searches}

The following 17 databases will be searched from inception to October 2014: MEDLINE (1946 to 4th week of October 2014), EMBASE (1980 to October 4 2014), the Cochrane Central Register of Controlled Trials (The Cochrane Library, 2014 Issue 10), the Cumulative Index to Nursing and Allied Health Literature (CINAHL, 1982 to October 2014), the Allied and Complementary Medicine Database (AMED, 1985 to October 2014), three Chinese databases (China National Knowledge Infrastructure (CNKI), the Chongqing VIP Chinese Science and Technology Periodical Database (VIP) and 
the Wanfang database), one Japanese database (Japan Science and Technology Information Aggregator, Electronic (J-STAGE)) and eight Korean databases (Korean Association of Medical Journal Edition (KAMJE), Korean Medical Database (KMBASE), Korean Studies Information Service System (KISS), National Discovery for Science Leaders (NDSL), Database Periodical Information Academic (DBpia), Korean National Assembly Digital Library (KNADL), Oriental Medicine Advanced Searching Integrated System (OASIS) and Korean Traditional Knowledge Portal (KTKP)). The WHO International Clinical Trials Registry Platform (ICTRP) will also be searched for ongoing and recently completed studies. The search terms consisted of three parts: laparoscopy (eg, laparoscopy, coelioscopy or video-assisted surgery), pain (eg, pain, analgesia or discomfort) and acupuncture (eg, acupuncture, electoracupuncture or auriculoacupuncture). The detailed search strategies for MEDLINE are presented in online supplementary appendix 1 .

\section{Searching other resources}

Bibliographic references in relevant publications (eg, anaesthesiology and pain medicine textbooks, other review articles and included clinical trials) will be manually searched to avoid missing eligible trials.

\section{Data collection and analysis}

Selection of studies

Three review authors (SL, JP and JK) will independently screen the titles and abstracts for potentially eligible studies identified by the searches. The authors will independently select and record their decisions on a standard eligibility form. If disagreements about the inclusion of a study cannot be resolved through discussion, the arbiter (J-DL) will make the final decision.

\section{Data extraction and management}

Three review authors (SL, JP and JK) will independently extract data using a standard data extraction form (eg, author, year of publication, country, study design, participants, condition, type of analgesics, acupuncture intervention, control intervention, outcome measures, main results and adverse events) after reading the full text of each article. Any disagreement regarding extracted data will be resolved by discussion or consultation among the reviewers. When the data are insufficient or ambiguous, we will contact the original study authors through email or telephone to request additional information.

\section{Assessment of risk of bias and reporting quality in included studies}

Three review authors (SL, JK and JP) will independently evaluate the risk of bias based on the Cochrane Collaboration's tool for assessing risk of bias of the included trials. The following domains will be assessed: (1) sequence generation; (2) allocation concealment; (3) blinding of participants; (4) blinding of outcome assessors; (5) incomplete outcome data; (6) selective outcome reporting and (7) other sources of bias (having factors that are likely to influence on results: (A) early cessation of trial due to apparent benefit or harm and (B) extreme baseline imbalance of age, comorbidity, duration of surgery, physical status or severity of condition before surgery). The risk of bias will be categorised into three levels: low, high and unclear risk of bias. Quality of reporting will be evaluated using the Consolidated Standards of Reporting Trials $(\text { CONSORT })^{23}$ and the revised STandards for Reporting Interventions in Clinical Trials of Acupuncture (STRICTA) ${ }^{24}$ The quality of reporting will be assessed using ' $\mathrm{Y}$ ' or ' $\mathrm{N}$ ': ' $\mathrm{Y}$ ' means the study reported the domains of CONSORT and STRICTA adequately; ' $N$ ' means the study did not report these domains adequately. ${ }^{25}$ Any disagreement will be resolved through discussion or consultation among the reviewers.

\section{Measures of treatment effect}

For continuous data, we will use the mean difference (MD), if the same methods or scales were used to measure the same outcome variable; if methods or scales were not the same, we will use the standardised MD with 95\% CIs. For dichotomous data, we will use the risk ratio to measure the treatment effect with $95 \%$ CIs. For ordinal data, we will convert the ordinal outcomes to dichotomous outcomes when the data need to be pooled.

\section{Unit of analysis issues}

When unit of analysis issues arise in studies that assessed pain repeatedly (at more than one time point), we will categorise the assessments into five different measurement points after laparoscopic surgery: (1) $4 \mathrm{~h}$, (2) $8 \mathrm{~h}$, (3) $24 \mathrm{~h}$, (4) up to $48 \mathrm{~h}$ and (5) more than $48 \mathrm{~h}$.

\section{Dealing with missing data}

Whenever possible, we will contact the original study authors to request for missing data. If the additional data cannot be obtained, we will analyse only the available data, and address the potential impact of the missing data in the discussion.

\section{Assessment of heterogeneity}

Heterogeneity among the included studies will be assessed by visual inspection of the forest plot and a $\chi^{2}$ test with a significance level of $\mathrm{p}<0.10$. $\mathrm{I}^{2}$ statistic will be calculated to quantify the inconsistencies among the included studies with a value of more than $50 \%$, indicating a meaningful heterogeneity. Heterogeneity of 0 $40 \%$ might not be important, $30-60 \%$ may be moderate, $50-90 \%$ may be substantial and $75-100 \%$ may be considerable heterogeneity. ${ }^{26}$

\section{Assessment of reporting biases}

When more than 10 studies are available, we will use visual asymmetry on a funnel plot to detect reporting bias, ${ }^{27}$ and Egger's regression test will be used to determine funnel plot asymmetry. ${ }^{28}$ 


\section{Data synthesis}

We will perform the meta-analysis using Review Manager software (RevMan, V.5.3 for Windows; the Nordic Cochrane Centre, Copenhagen, Denmark). A random effects model with $95 \%$ CIs will be used in the calculation of the pooled treatment-effect estimates, since substantial heterogeneity is expected among studies that will be included in this review. We will not attempt meta-analysis if considerable heterogeneity $\left(\mathrm{I}^{2}>75 \%\right)$ cannot be explained by the clinical and methodological diversity. ${ }^{27}$ When trials have more than two acupuncture groups with different stimulation styles (eg, high or low electrical stimulation) or points (eg, local or distal acupuncture points), meta-analysis will be conducted in careful consideration of each group. In our trial, we will combine the data of the acupuncture groups and compare the merged acupuncture group with the control. ${ }^{29}$ In this case, we will also perform a sensitivity analysis in which each acupuncture group will be compared individually with control groups, divided roughly into equal halves to avoid double counting of data in the control group in the meta-analysis.

\section{Subgroup analysis and investigation of heterogeneity}

When sufficient numbers of studies are available, subgroup analysis will be conducted to interpret the heterogeneity among studies according to the following:

1. Type of acupuncture treatment (eg, manual acupuncture, electroacupuncture or ear acupuncture)

2. Timing of acupuncture treatment (eg, before surgery, during surgery, after surgery or a combination thereof)

3. Type of control (eg, no treatment/waitlist, placebo/ sham acupuncture, active treatment or add-on effect for active control)

4. Duration of follow-up $(4,8,24$, up to or more than $48 \mathrm{~h})$.

\section{Sensitivity analysis}

When sufficient numbers of studies are available, sensitivity analysis will be performed to identify whether the results are robust in the review according to the following:

1. Methodological qualities (eg, whether sequence generation and allocation concealment are adequately conducted or not)

2. Sample size (eg, less or more than 40 participants in each group $)^{30}$

3. Analysis issues in trials having more than two acupuncture groups (ie, comparing the merged acupuncture group vs each acupuncture group separately with the control group in meta-analysis).

\section{Summary of evidence}

We will summarise the results of the main outcomes (primary outcomes and adverse events) in 'Summary of findings' tables. The quality of evidence in the main outcomes will be assessed using the Grading of Recommendations Assessment, Development and Evaluation
(GRADE) approach considering the following factors: (1) limitations in the design and implementation; (2) indirectness of evidence; (3) unexplained heterogeneity or inconsistency of results; (4) imprecision of results; (5) high probability of publication bias. We will categorise the quality of evidence into four levels: high, moderate, low and very low quality. ${ }^{27}$

\section{DISCUSSION}

The object of this SR is to assess the effectiveness and safety of acupuncture treatment in postoperative pain after laparoscopic surgery. Recently, two SRs of acupuncture for postoperative pain were published. ${ }^{9}{ }^{10}$ Although the type of surgery may influence the pain site and intensity, both reviews included multiple surgery types such as abdominal, lumbar or dental surgery as well as laparoscopic surgery. ${ }^{31}$ Moreover, the definition of acupuncture used in those studies is either too broad, including multiple types of acupuncture point stimulation such as acupressure and transcutaneous electrical acupoint stimulation, ${ }^{9}$ or too narrow, only focusing on ear acupuncture. ${ }^{10}$ Therefore, we will evaluate postoperative pain induced by laparoscopic surgery and define acupuncture treatment adequately as two components: (1) needling with penetration of the skin and (2) on specific points including traditional acupuncture points or painful points around the incision or shoulder region.

This SR will provide a summary of the current evidence on the effectiveness and safety of acupuncture for patients with postoperative pain after laparoscopic surgery. This evidence will provide information useful to patients, practitioners and health policy makers who consider acupuncture a potential adjuvant therapy to conventional analgesics.

Contributors The search strategy was developed by SL. SL, JP and JK will search and select the studies. J-DL will act as an arbiter in the selection stage. Extraction of data, assessment of risk of bias, and reporting quality and quality of evidence will be performed by SL, JP and JK. Interpretation of the analyses will be performed by all authors. All authors read and approved the final manuscript for publication.

\section{Competing interests None.}

Provenance and peer review Not commissioned; externally peer reviewed.

Open Access This is an Open Access article distributed in accordance with the Creative Commons Attribution Non Commercial (CC BY-NC 4.0) license, which permits others to distribute, remix, adapt, build upon this work noncommercially, and license their derivative works on different terms, provided the original work is properly cited and the use is non-commercial. See: http:// creativecommons.org/licenses/by-nc/4.0/

\section{REFERENCES}

1. Yoo J. Laparoscopic colorectal surgery. Perm J 2008;12:27-31

2. Li L, Tian J, Tian $\mathrm{H}$, et al. The efficacy and safety of different kinds of laparoscopic cholecystectomy: a network meta analysis of 43 randomized controlled trials. PLOS ONE 2014;9:e90313.

3. Bisgaard T, Kehlet H, Rosenberg J. Pain and convalescence after laparoscopic cholecystectomy. Eur J Surg 2001;167:84-96.

4. Patel HR, Linares A, Joseph JV. Robotic and laparoscopic surgery: cost and training. Surg Oncol 2009;18:242-6.

5. Tsai HW, Chen YJ, Ho CM, et al. Maneuvers to decrease laparoscopy-induced shoulder and upper abdominal pain: a randomized controlled study. Arch Surg 2011;146:1360-6. 
6. Mayor DF. Electroacupuncture: a practical manual and resource. Edinburgh, New York: Churchill Livingstone Elsevier, 2007.

7. World Health Organization. WHO international standard terminologies on traditional medicine in the Western Pacific Region. Manila, Philippines: World Health Organization, Western Pacific Region, 2007.

8. Vickers AJ, Cronin AM, Maschino AC, et al. Acupuncture for chronic pain: individual patient data meta-analysis. Arch Intern Med 2012:172:1444-53.

9. Sun Y, Gan TJ, Dubose JW, et al. Acupuncture and related techniques for postoperative pain: a systematic review of randomized controlled trials. Br J Anaesth 2008;101:151-60.

10. Usichenko TI, Lehmann C, Ernst E. Auricular acupuncture for postoperative pain control: a systematic review of randomised clinical trials. Anaesthesia 2008;63:1343-8.

11. White A, Cummings TM, Filshie J. An introduction to western medical acupuncture. Edinburgh, New York: Churchill Livingstone Elsevier, 2008.

12. Carlsson C. Acupuncture mechanisms for clinically relevant long-term effects-reconsideration and a hypothesis. Acupunct Med 2002;20:82-99.

13. Silva JR, Silva ML, Prado WA. Analgesia induced by $2-$ or $100-\mathrm{Hz}$ electroacupuncture in the rat tail-flick test depends on the activation of different descending pain inhibitory mechanisms. J Pain 2011;12:51-60.

14. Han JS. Acupuncture and endorphins. Neurosci Lett 2004;361:258-61.

15. Lillemoe KD, Lin JW, Talamini MA, et al. Laparoscopic cholecystectomy as a "true" outpatient procedure: initial experience in 130 consecutive patients. $J$ Gastrointest Surg 1999;3:44-9.

16. Guillotreau J, Game X, Mouzin M, et al. Radical cystectomy for bladder cancer: morbidity of laparoscopic versus open surgery. $J$ Urol 2009;181:554-9.

17. Hutchison R, Chon EH, Tucker JW, et al. A comparison of a fentanyl, morphine, and hydromorphone patient-controlled intravenous delivery for acute postoperative analgesia: a multicenter study of opioid-induced adverse reactions. Hosp Pharm 2006;41:659-63.

18. Rawal N. Analgesia for day-case surgery. $\mathrm{Br} J$ Anaesth 2001;87:73-87.
19. Lovatsis D, Jose JB, Tufman A, et al. Assessment of patient satisfaction with postoperative pain management after ambulatory gynaecologic laparoscopy. J Obstet Gynaecol Can 2007;29: 664-7.

20. American Society of Anesthesiologists Task Force on Acute Pain Management. Practice guidelines for acute pain management in the perioperative setting: an updated report by the American Society of Anesthesiologists Task Force on Acute Pain Management. Anesthesiology 2012;116:248-73.

21. Mouton WG, Bessell JR, Otten KT, et al. Pain after laparoscopy. Surg Endosc 1999;13:445-8.

22. Bisgaard T, Klarskov B, Rosenberg J, et al. Characteristics and prediction of early pain after laparoscopic cholecystectomy. Pain 2001;90:261-9.

23. Schulz KF, Altman DG, Moher D, et al. CONSORT 2010 statement: updated guidelines for reporting parallel group randomized trials. Ann Intern Med 2010;152:726-32.

24. MacPherson H, Altman DG, Hammerschlag R, et al. Revised STandards for Reporting Interventions in Clinical Trials of Acupuncture (STRICTA): extending the CONSORT statement. $J$ Altern Complement Med 2010;16:ST1-14.

25. Kim KH, Kang JW, Lee MS, et al. Assessment of the quality of reporting in randomised controlled trials of acupuncture in the Korean literature using the CONSORT statement and STRICTA guidelines. BMJ Open 2014;4:e005068.

26. Higgins JP, Thompson SG. Quantifying heterogeneity in a meta-analysis. Stat Med 2002;21:1539-58.

27. Higgins JPT, Green S. Cochrane Handbook for Systematic Reviews of Interventions Version 5.1.0 [updated March 2011]. The Cochrane Collaboration, 2011. http://www.cochrane-handbook.org

28. Egger M, Davey SG, Schneider M, et al. Bias in meta-analysis detected by a simple, graphical test. BMJ 1997;315:629-34.

29. Littell JH, Corcoran J, Vijayan P. Systematic reviews and meta-analysis. Oxford, New York: Oxford University Press, 2008

30. Moore RA, Gavaghan D, Tramer MR, et al. Size is everything-large amounts of information are needed to overcome random effects in estimating direction and magnitude of treatment effects. Pain 1998;78:209-16.

31. Bidese BL, Sakuma KA, Andrade Júnior Ad, et al. Postoperative analgesia by non-specialists in pain. Rev Dor 2014;15:36-40. 